\title{
Errata: A Simple Theoretical Analysis of the Low- Energy Spin Fluctuation Peak Observed by Neutrons in Liquid ${ }^{3} \mathrm{He}^{*}$
}

\author{
M. T. Béal-Monod \\ Laboratoire de Physique des Solides, Université Paris-Sud, Orsay, France
}

(Received September 26, 1979)

1. Formula (30) should read

$$
S_{i}(q)=\int S_{i}(q, \omega) d(\hbar \omega)
$$

2. The values of $\bar{p}_{1}$ in (35) should read, respectively,

$$
\begin{array}{lll}
\bar{p}_{1}=0.9775 & \text { for } & \bar{I}=0.891 \\
\bar{p}_{1}=1.1134 & \text { for } & \bar{I}=0.958
\end{array}
$$

as following the straightforward algebra involved in (34). 